DOI: $10.4274 /$ gulhane.galenos.2021.35744

Gulhane Med J 2022;64:67-72

\title{
The effects of urinary incontinence on quality of life and sexual function in women of reproductive age
}

\author{
(D) Ayçağ Yorgancı, (1) Könül Mürsel, (1) Nezaket Kadıoğlu, (1) Şule Özel, (1) Özlem Evliyaoğlu Bozkurt, \\ (1) Yaprak Engin Üstün
}

University of Health Sciences Turkey, Ankara Dr. Zekai Tahir Burak Women's Health Training and Research Hospital, Clinic of Obstetrics and Gynecology, Ankara, Turkey

Date submitted:

08.02.2021

Date accepted:

27.03.2021

Online publication date:

15.03.2022

\section{Corresponding Author:}

Ayçağ Yorgancı, M.D., University of Health Sciences Turkey, Ankara Dr.

Zekai Tahir Burak Women's Health

Training and Research Hospital, Clinic of Obstetrics and Gynecology, Ankara, Turkey

aycagyorganci@gmail.com

ORCID:

orcid.org/0000-0003-2565-6789

Keywords: Female urinary incontinence, quality of life, sexual function, PISQ-12

\begin{abstract}
Aims: Urinary incontinence (UI) is a common condition in women and might affect women's physical and psychological health in different aspects. This study evaluated the effects of UI on the quality of life (QoL) and sexual function in women of reproductive age.
\end{abstract}

Methods: In this prospective case-control study, sexually active and premenopausal women who were admitted to the urogynecology outpatient clinic of our hospital with UI were included. Women without UI complaints were selected for the control group. All the women were asked to fill the Urogenital Distress Inventory-6 (UDI-6), the Incontinence Impact Questionnaire-7 (IIQ-7), and Pelvic Organ Prolapse/Urinary Incontinence Sexual Function Questionnaire-12 (PISQ-12).

Results: There were 64 women [median age: 42 (12)] in the UI group and 58 women [median age: 40 (11)] in the control group. Although the median age was similar in the two groups, the UI group had significantly more gravidas [3 (1) vs. 2 (1); $\mathrm{p}<0.00$ ] and parity [2.5 (1) vs. 2 (1); $p<0.001]$, body mass index [27.9 (7.2) vs. 24.9 (5.1); $p=0.002]$, and Pelvic Organ ProlapseQuantification [0 (0) vs. $0(0) ; p=0.03$ ] levels. The most common UI type was mixed UI (64.1\%) followed by the stress (20.3\%) and urge UI (15.6\%). The UI group had significantly higher scores in both the subscale and total scores in IIQ-7 and UDI-6 questionnaires $(p<0.001)$. For the PSIQ-12 questionnaire, the behavioral-emotive subscale did not differ between the two groups, while physical and partner subscales and the total score were significantly lower in the UI group $(p<0.001)$.

Conclusions: This study showed that UI had a negative impact on QoL and sexual function in women of reproductive age. Sexual dysfunction occurs mainly on physical and partner-related issues.

\section{Introduction}

Urinary incontinence (UI), involuntary leakage of urine, is a common disorder observed in women. The incidence of UI in females increases by age and it is found in $12 \%$ in nulligravid women around the age of 20 , and $45-78 \%$ in women living in nursing homes $(1,2)$. Aging, obesity, parity, mode of delivery, and menopause are major risk factors for female UI (3-6). Apart from the disturbing physical effects of wetness, UI in women has various psychological negative effects such as anxiety, depression, social isolation, decreased quality of life (QoL), and sexual dysfunction (7-9).
$\mathrm{UI}$ is divided into three subheadings: stress incontinence, urge incontinence, and mixed incontinence (10). While stress incontinence is defined as involuntary $\mathrm{UI}$ with laughing, coughing, sneezing, or any similar efforts increasing intraabdominal pressure, urge incontinence is defined as leakage of urine with a sudden desire for urination due to involuntary detrusor contractions. Mixed incontinence is involuntary urinary leakage associated with both conditions

Coital incontinence is defined as urinary leakage that occurs during sexual intercourse (10). It is generally accepted to occur in two different ways. Coital incontinence during penetration 
or intercourse is associated with stress UI (11), while coital incontinence during orgasm is usually associated with detrusor overactivity $(11,12)$. Coital incontinence has been reported in about 1 in 3 incontinent women (13). In a recent international study, the rate of coital incontinence was reported as $8 \%$ at penetration, $9 \%$ at orgasm, and $35 \%$ during intercourse (14). Intriguingly, nearly half of the patients reported a combination of them.

Regardless of the underlying mechanism, $\mathrm{UI}$ could lead to sexual dysfunction in women (15). In this study, we measured the effects of UI on QoL and sexual dysfunction, specifically in women of reproductive age without significant pelvic organ prolapse.

\section{Methods}

In this prospective case-control study, sexually active and premenopausal women who were admitted to Urogynecology Outpatient Clinic of Ankara Zekai Tahir Burak Women's Health Training and Research Hospital between August 2018 and March 2019 with the complaint of UI were included in the study. The study was approved by the Ethics Committee of the Ankara Zekai Tahir Burak Women's Health Training and Research Hospital (protocol number: 07/2018, date: 13.12.2018). For the control group, women who were admitted to the gynecology outpatient clinics of the hospital without any $\mathrm{UI}$ complaints were selected. The inclusion criteria were age 18 or older, being sexually active for the previous six months, and being in the premenopausal period. Women who had physical or psychiatric diseases that required a significant medical intervention in the previous year, a history of previous prolapse-incontinence surgery or current pelvic organ prolapse higher than stage 1 , chronic pelvic pain, difficulties in reading and understanding the Turkish language, and women using hormonal contraception were excluded. All participants signed informed consent before participating in the study.

The women who were admitted to the urogynecology clinic with UI were evaluated according to the guidelines (16). The $\mathrm{UI}$ symptoms and as well as systemic symptoms for urinary tract infections, dysuria, and hematuria were questioned by the standard urogynecology application form of the hospital. Urinalysis and culture were obtained. The cough stress test, $Q$ tip test, residual volume were assessed and the presence of pelvic organ prolapse was evaluated according to the Pelvic Organ Prolapse-Quantification (POP-Q) classification system. Urodynamic evaluation was done in complicated cases (16). Incontinent women were classified as stress $\mathrm{UI}$, urge $\mathrm{UI}$, or mixed UI according to the symptoms, clinical and urodynamic findings (if performed).

We used the Incontinence Impact Questionnaire-7 (IIQ7) and the Urogenital Distress Inventory-6 (UDI-6) to evaluate the effects of UI on QoL, and Pelvic Organ Prolapse/Urinary
Incontinence Sexual Function Questionnaire-12 (PISQ-12) to evaluate its effects on sexual dysfunction. The IIQ-7 and UDI-6 were developed and combined to assess the impact of $\mathrm{UI}$ on QoL (17). The IIQ-7 consists of seven questions with the subscales of physical, travel, social and emotional (17). The response to each question measures the severity of the symptoms by grading the responses from zero (not at all) to three (greatly) points. The total score and subscale scores are converted to a range between 0 and 100. Higher scores represent a greater negative impact on QoL. The UDI-6 consists of six questions with three subscales addressing irritation symptoms, stress symptoms, and obstructive/voiding difficulty symptoms (17). The scoring is similar to IIQ-7. Similarly, higher scores indicate more severe symptoms and increased symptom distress. Both IIQ-7 and UDI-6 have been validated for the Turkish female population (18).

The PISQ-12 is a Likert-type questionnaire with 12 questions. It is specifically designed to evaluate the effect of pelvic organ prolapse/UI on female sexual function (19). It consists of three subscales: behavioral/emotive, physical, and partner-related factors. The maximum total score that can be obtained is 48 and higher total and subscale scores are associated with better sexual functioning. Its validity and reliability in the Turkish version were demonstrated by Cam et al. (20).

\section{Statistical Analysis}

Statistical Package for Social Sciences Statistics for Windows, version 22.0 (Armonk, NY: IBM Corp., 2013 ) was used for the analysis of the data. The suitability of the data to a normal distribution was examined by the KolmogorovSmirnov test. Parametric methods were used in the analysis of variables with normal distribution and non-parametric methods were used in the analysis of variables that did not have a normal distribution. Categorical data are expressed as numbers ( $\mathrm{n}$ ) and percentages (\%). Normally distributed nominal data are shown in tables as mean \pm standard deviation, while non-normally distributed nominal data are expressed as the median \pm interquartile range (IQR: 25-75). Cross tables were created for the necessary questions. The chi-square test, Mann-Whitney $U$ test, and independent sample test were used for comparative statistical analysis. Spearman correlation test was used to examine the correlations of variables with each other. The data were analyzed at a 95\% confidence level and the $p$-value was accepted as significantly less than 0.05 . Before the study was begun, the statistical program available on the British Colombia University Department of Statistics website was used to calculate the power analysis of the study (https:// www.stat.ubc.ca/ rollin/stats/ssize/n2). With a two-sided test, it was found that 49 patients in each group were sufficient to reach an alpha $=0.05$ significance level and achieve a power of at least $95 \%(20)$. 


\section{Results}

During the study period, there were 129 women who consented to participate in the study. Four women in the UI group and three women in the control group were excluded due to incomplete filling of the forms. Therefore, 64 women in the UI group and 58 women in the control group were included in the analysis (Figure 1). The median age of the total participants was 41 (IQR: 12). The comparison of the demographic characteristics of the two groups is given in Table 1. While there was not any statistical significance between the median ages of the groups, the UI group had a statistically significantly higher number of gravida and parity, body mass index (BMI), and POP-Q levels. Although the median and IQR values of the POP-Q levels were similar between the groups, there was a statistically significant

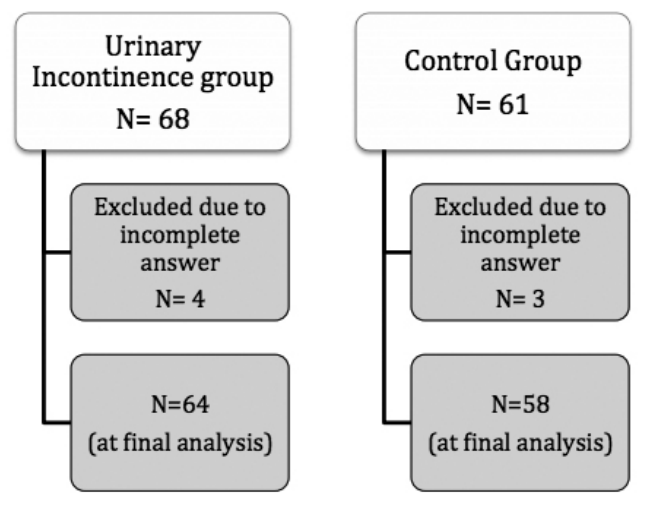

Figure 1. Flow-chart of the study participants difference due to the distribution within the groups $(p=0.030)$. The most common UI type was mixed UI $(64.1 \%)$ followed by stress UI (20.3\%) and urge UI (15.6\%).

The comparison of the subscale and total scores of the IIQ7, UDI-6, and PISQ-12 between the groups is shown in Table 2. The UI group had statistically significantly higher in both the subscale and total scores in IIQ-7 and UDI-6 questionnaires $(p<0.001)$. For the PSIQ-12 questionnaire, the behavioralemotive subscale did not differ between the two groups $(p=0.31)$, while physical and partner subscales and the total scores were statistically significantly lower in the UI group $(p<0.001)$.

The correlations between the total PISQ-12 score and the IIQ-7 and UDI-6 subscale and total scores, age, gravida, parity, $\mathrm{BMI}$, and POP-Q stage were evaluated. The total PISQ-12 score was negatively correlated with all listed variables except age. The behavior-emotive subscale score was negatively correlated with only the number of gravidities $(r=-0.270, p=0.005)$ and parity $(r=-0.210, p=0.030)$.

\section{Discussion}

Our results showed that the UI has a significant effect on QoL and sexual function in women of reproductive age without pelvic organ prolapse. UI decreases QoL and sexual function in women due to its physical and psychological effects. While the damaging effects of $\mathrm{UI}$ were seen in the subscale and total scores of UDI-6 and IIQ-7, the PISQ-12 questionnaire results warrant further evaluation.

\begin{tabular}{|c|c|c|c|}
\hline & Incontinence group $(n=64)$ & Control group ( $n=58$ ) & p \\
\hline Age, median (IQR) & $42(12)$ & $40(11)$ & 0.300 \\
\hline Gravidity, median (IQR) & $3(1)$ & $2(1)$ & $<0.001$ \\
\hline Parity, median (IQR) & $2.5(1)$ & $2(1)$ & $<0.001$ \\
\hline \multicolumn{4}{|l|}{ Delivery mode, $\mathbf{n}(\%)$} \\
\hline Nil & $1(1.6)$ & $1(1.7)$ & \\
\hline Vaginal & $51(79.7)$ & $40(69)$ & \\
\hline $\mathrm{C} / \mathrm{S}$ & $10(15.6)$ & $15(25.9)$ & 0.560 \\
\hline Vaginal $+\mathrm{C} / \mathrm{S}$ & $2(3.1)$ & $2(3.4)$ & \\
\hline BMI & $27.9(7.2)$ & $24.9(5.1)$ & 0.002 \\
\hline \multicolumn{4}{|l|}{ Medical diseases, $\mathbf{n}(\%)$} \\
\hline None & 39 (60.9) & $47(81.0)$ & \\
\hline Hypertension & $3(4.6)$ & $0(0)$ & \\
\hline Diabetes mellitus & $11(17.1)$ & $4(6.8)$ & \\
\hline COPD & $7(10.9)$ & $4(6.8)$ & 0.250 \\
\hline Other & $4(6.2)$ & $3(5.1)$ & \\
\hline POP-Q & $0(0)$ & $0(0)$ & 0.030 \\
\hline \multicolumn{4}{|l|}{ Type of UI, $\mathbf{n}(\%)$} \\
\hline Stress & $13(20.3)$ & & \\
\hline Urge & $10(15.6)$ & NA & \\
\hline Mixed & $41(64.1)$ & & \\
\hline
\end{tabular}




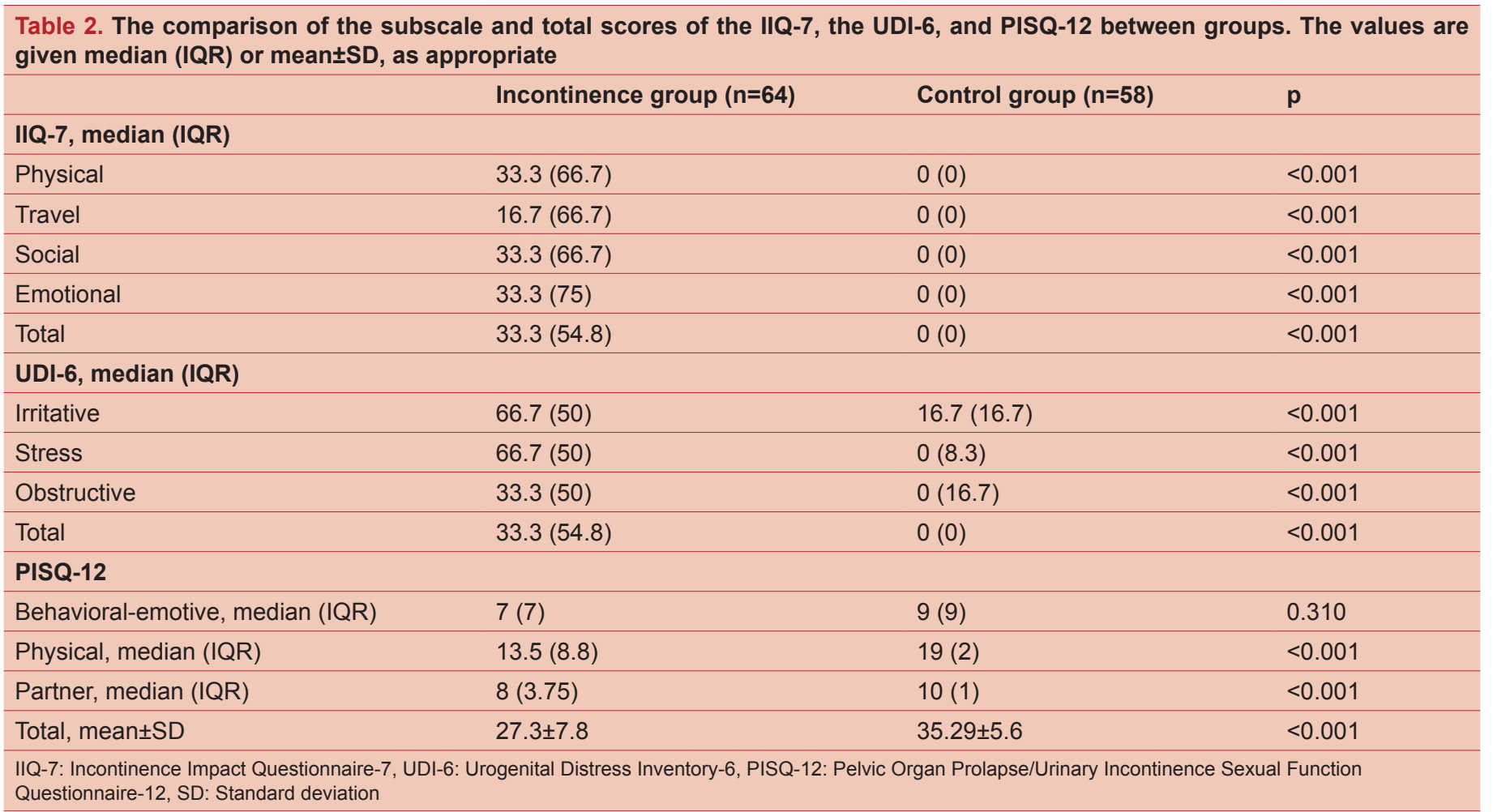

The behavioral and emotive subscale of the PISQ-12 measures women's desire, orgasm, sexual excitement, and satisfaction from sexual activity. According to our findings, this subscale did not differ between the continent and incontinent women. However, the physical subscale questioning dyspareunia, UI during sexual intercourse, restriction of sexual activity because of $\mathrm{UI}$ or pelvic organ prolapse, and execration, embarrassment, feeling guilty from sexual activity, were significantly lower in the incontinent women. The partner scale, which explores erection problems and premature ejaculation in the partner and intensity of orgasm degree in the last 6 months in women, was also significantly lower in the incontinent women. Thus, our results suggest that $\mathrm{UI}$ does not affect sexual arousal and orgasm among women of reproductive age. The low total PISQ-12 score was mainly due to the low physical and partner subscale scores. Although low physical subscale scores in incontinent women are an expected result, low partner subscale scores are difficult to explain. It is much more difficult for us to speculate whether it is due to urinary leakage of women during sexual intercourse. Indeed, Bekker et al. (21) investigated partners of incontinent women, and their results were in accordance with our study. Men who have partners with $\mathrm{UI}$ showed worse total sexual scores together with worse scores in frequency, satisfaction, and erectile dysfunction subscales.

Female sexuality is a complex condition that is influenced by cultural, religious, emotional, and physical factors and depends on the quality of the relationship rather than quantity. As obstetrics and gynecology professionals, we focus almost entirely on the female patient and try treating her. However, as can be seen in our study results, we may overlook that the partner indirectly affected using this process is also an important part of the problem. We can speculate that this problem may be more important in closed societies than in developed Western societies. Based on the results of the study, it would be an appropriate approach to at least include the partner in the treatment process and to provide counseling when necessary. With another study that can be planned in the future, it may be possible to examine the way the partner perceives this disease and the behavioral problems that occur as a result.

The reason we did not find a difference between behavioralemotive subscale scores of those with and without UI might be the exclusion of menopausal women in our study. Female external genitalia, vagina, bladder trigone, and urethra exhibit estrogen receptors. The term "genitourinary syndrome of menopause" is currently used to define the effects of low estrogen levels on the genitourinary epithelium (22). The symptoms of the genitourinary syndrome of menopause are genital symptoms of vaginal dryness, burning, and irritation; sexual symptoms of lack of lubrication, discomfort or pain, and impaired function; and urinary symptoms of urgency, dysuria, and recurrent urinary tract infections $(22,23)$. Besides, sexual desire and arousal are declined in postmenopausal women $(24,25)$. Thus, this might explain the controversy between our results and others $(20,26)$. However, various validated questionnaires are used to evaluate sexual function with different subscales. Although negative impacts of UI on sexual function are general findings, subscale 
evaluations differ substantially due to the variability of the questionnaires (14,27-29). For instance, in a recent study from our country conducted in a similar cohort of women like our study, the frequency, satisfaction, avoidance, and anorgasmia sexual subdomains were significantly worse in the UI group compared to the control group (28). However, partner-related factors were not sought by this questionnaire. Consistent with the previously reported studies, our findings showed that incontinent women had higher gravidity, parity, BMI, and POP-Q stage compared to continent women. Besides, negative correlations were observed between sexual function and these factors.

Another important point to consider is that different subtypes of UI have varying effects on sexual function. The mixed UI subtype affects sexual function worse than other subtypes $(28,30,31)$. Similarly, mixed UI has affected QoL negatively more than other subtypes $(28,31,32)$. As most of our patients were in the mixed UI group, we could not investigate the effects of different types of UI on QoL and sexual function separately.

There are strengths and limitations of our study. Unlike other studies, we have specifically addressed women of reproductive age and underlined their most affected sexual dysfunction. However, our study was conducted in a single center with a relatively small number of participants. Furthermore, we did not assess the effects of different types of UI. Although we have evaluated pelvic organ prolapse, we did not specifically address anterior and posterior compartment defects.

\section{Conclusion}

In conclusion, UI has negative impacts on QoL and sexual function in women in the reproductive period. This negative effect is mainly observed in the physical and partner-related aspects of the sexual relationship. Accordingly, it is far most important for women's health to question women who apply to gynecology outpatient clinics and provide them with counseling about preventive and therapeutic approaches.

\section{Ethics}

Ethics Committee Approval: The study were approved by the Ankara Zekai Tahir Burak Women's Health Training and Research Hospital of Ethics Committee (protocol number: 07/2018, date: 13.12.2018).

Informed Consent: Consent form was filled out by all participants.

Peer-review: Externally peer-reviewed.

\section{Authorship Contributions}

Surgical and Medical Practices: A.Y., K.M., N.K., Concept: A.Y., Ş.Ö., Ö.E.B., Y.E.Ü., Design: A.Y., Ş.Ö., Ö.E.B., Y.E.Ü., Data Collection or Processing: A.Y., K.M., N.K., Analysis or Interpretation: A.Y., Literature Search: A.Y., K.M., N.K., Ş.Ö., Writing: A.Y., Ö.E.B.
Conflict of Interest: No conflict of interest was declared by the authors.

Financial Disclosure: The authors declared that this study received no financial support.

\section{References}

1. O'Halloran T, Bell RJ, Robinson PJ, Davis SR. Urinary incontinence in young nulligravid women: a cross-sectional analysis. Ann Intern Med. 2012;157:87-93.

2. Offermans MP, Du Moulin MF, Hamers JP, Dassen T, Halfens RJ. Prevalence of urinary incontinence and associated risk factors in nursing home residents: a systematic review. Neurourol Urodyn. 2009;28:288-294.

3. MacLennan AH, Taylor AW, Wilson DH, Wilson D. The prevalence of pelvic floor disorders and their relationship to gender, age, parity and mode of delivery. BJOG. 2000;107:1460-1470.

4. Fritel X, Fauconnier A, Levet C, Benifla JL. Stress urinary incontinence 4 years after the first delivery: a retrospective cohort survey. Acta Obstet Gynecol Scand. 2004;83:941-945.

5. Al-Mukhtar Othman J, Akervall S, Milsom I, Gyhagen M. Urinary incontinence in nulliparous women aged 25-64 years: a national survey. Am J Obstet Gynecol. 2017;216:149.

6. Akdemir Y NK, Büyükuysal Ç, Özmen Ü, Harma M, Harma Mi. Comparison of Quality of Life and Sexual Functions of Obese and Non-Obese Women with Urinary Incontinence. Turkish Journal of Diabetes and Obesity. 2020;2:84-89.

7. Coyne KS, Sexton CC, Irwin DE, Kopp ZS, Kelleher CJ, Milsom I. The impact of overactive bladder, incontinence and other lower urinary tract symptoms on quality of life, work productivity, sexuality and emotional well-being in men and women: results from the EPIC study. BJU Int. 2008;101:13881395.

8. Yip SK, Cardozo L. Psychological morbidity and female urinary incontinence. Best Pract Res Clin Obstet Gynaecol. 2007;21:321-329.

9. Orhan C, Ozgul S, Baran E, et al. The Effect of Incontinence Severity on Symptom Distress, Quality of Life, and Pelvic Floor Muscle Function in Turkish Women with Urinary Incontinence. Gynecology Obstetrics \& Reproductive Medicine. 2020;26:51-57.

10. Haylen BT, de Ridder D, Freeman RM, et al. An International Urogynecological Association (IUGA)/International Continence Society (ICS) joint report on the terminology for female pelvic floor dysfunction. Int Urogynecol J. 2010;21:5-26.

11. Madhu C, Hashim H, Enki D, Yassin M, Drake M. Coital incontinence: what can we learn from urodynamic assessment? Urology. 2015;85:1034-1038.

12. Serati M, Salvatore S, Uccella S, et al. Urinary incontinence at orgasm: relation to detrusor overactivity and treatment efficacy. Eur Urol. 2008;54:911-915.

13. Demir O, Comba C. Investigation of the Incidence of Coital Incontinence in Incontinent Women. Gynecology Obstetrics \& Reproductive Medicine. 2020;26:130-133. 
14. Illiano E, Mahfouz W, Giannitsas K, et al. Coital Incontinence in Women With Urinary Incontinence: An International Study. J Sex Med. 2018;15:1456-162.

15. Mota RL. Female urinary incontinence and sexuality. Int Braz J Urol. 2017;43:20-28.

16. No authors listed. Committee Opinion No. 603: Evaluation of uncomplicated stress urinary incontinence in women before surgical treatment. Obstet Gynecol. 2014;123:1403-1407.

17. Uebersax JS, Wyman JF, Shumaker SA, McClish DK, Fantl JA. Short forms to assess life quality and symptom distress for urinary incontinence in women: the Incontinence Impact Questionnaire and the Urogenital Distress Inventory. Continence Program for Women Research Group. Neurourol Urodyn. 1995;14:131-139.

18. Cam C, Sakalli M, Ay P, Cam M, Karateke A. Validation of the short forms of the incontinence impact questionnaire (IIQ7 ) and the urogenital distress inventory (UDI-6) in a Turkish population. Neurourol Urodyn. 2007;26:129-133.

19. Rogers RG, Coates KW, Kammerer-Doak D, Khalsa S, Qualls C. A short form of the Pelvic Organ Prolapse/Urinary Incontinence Sexual Questionnaire (PISQ-12). Int Urogynecol J Pelvic Floor Dysfunct. 2003;14:164-168.

20. Cam C, Sancak P, Karahan N, Sancak A, Celik C, Karateke A. Validation of the short form of the Pelvic Organ Prolapse/ Urinary Incontinence Sexual Questionnaire (PISQ-12) in a Turkish population. Eur J Obstet Gynecol Reprod Biol. 2009;146:104-107.

21. Bekker MD, Beck JJ, Putter H, et al. Sexual experiences of men with incontinent partners. J Sex Med. 2010;7:1877-1882.

22. Portman DJ, Gass ML; Vulvovaginal Atrophy Terminology Consensus Conference Panel. Genitourinary syndrome of menopause: new terminology for vulvovaginal atrophy from the International Society for the Study of Women's Sexual Health and the North American Menopause Society. Menopause. 2014;21:1063-1068.
23. Palacios S, Mejia A, Neyro JL. Treatment of the genitourinary syndrome of menopause. Climacteric. 2015;18(Suppl 1):2329.

24. Leiblum SR, Koochaki PE, Rodenberg CA, Barton IP, Rosen RC. Hypoactive sexual desire disorder in postmenopausal women: US results from the Women's International Study of Health and Sexuality (WISHeS). Menopause. 2006;13:46-56.

25. Avis NE, Stellato R, Crawford S, Johannes C, Longcope C. Is there an association between menopause status and sexual functioning? Menopause. 2000;7:297-309.

26. Eroğlu A, Aydın M, Onuk Ö, Çilesiz NC, Nuhoğlu B. Investigation of Sexual Dysfunction in Premenopausal Women with Urinary Incontinence. J Acad Res Med. 2020;10:160-165.

27. Nilsson $M$, Lalos $\mathrm{O}$, Lindkvist $\mathrm{H}$, Lalos $\mathrm{A}$. How do urinary incontinence and urgency affect women's sexual life? Acta Obstet Gynecol Scand. 2011;90:621-628.

28. Dogan K, Vural M, Akyüz F. Evaluation of effects of urinary incontinence subtypes on women's sexual function using the Golombok-Rust Inventory of Sexual Satisfaction. J Obstet Gynaecol Res. 2017;43:551-556.

29. Su CC, Sun BY, Jiann BP. Association of urinary incontinence and sexual function in women. Int J Urol. 2015;22:109-113.

30. Coksuer H, Ercan CM, Haliloglu B, et al. Does urinary incontinence subtype affect sexual function? Eur J Obstet Gynecol Reprod Biol. 2011;159:213-217.

31. Bilgic D, Kizilkaya Beji N. How do urinary incontinence types affect sexual function and quality of life for Turkish women? Low Urin Tract Symptoms. 2020;12:253-259.

32. Caruso S, Brescia R, Matarazzo MG, Giunta G, Rapisarda AMC, Cianci A. Effects of Urinary Incontinence Subtypes on Women's Sexual Function and Quality of Life. Urology. 2017;108:59-64. 